\title{
Maria Chiara Gnocchi, Le Parti pris des périphéries. Les "Prosateurs français contemporains" des éditions Rieder (1921-1939)
}

\section{Riccardo Renedettini}

\section{(2) OpenEdition Journals}

\section{Edizione digitale}

URL: http://journals.openedition.org/studifrancesi/8356

DOI: $10.4000 /$ studifrancesi.8356

ISSN: 2421-5856

\section{Editore}

Rosenberg \& Sellier

\section{Edizione cartacea}

Data di pubblicazione: 1 mai 2009

Paginazione: 205-206

ISSN: 0039-2944

\section{Notizia bibliografica digitale}

Riccardo Renedettini, «Maria Chiara Gnocchi, Le Parti pris des périphéries. Les "Prosateurs français contemporains" des éditions Rieder (1927-1939)», Studi Francesi [Online], 157 (LIII | I) | 2009, online dal 30 novembre 2015, consultato il 13 janvier 2021. URL: http://journals.openedition.org/studifrancesi/8356 ; DOI: https://doi.org/10.4000/studifrancesi.8356

Questo documento è stato generato automaticamente il 13 janvier 2021.

\section{cc) (†) $\odot$}

Studi Francesi è distribuita con Licenza Creative Commons Attribuzione - Non commerciale - Non opere derivate 4.0 Internazionale. 


\title{
Maria Chiara Gnocchi, Le Parti pris des périphéries. Les "Prosateurs français contemporains" des éditions Rieder (1921-1939)
}

\author{
Riccardo Renedettini
}

\section{NOTIZIA}

MARIA CHIARA GNOCCHI, Le Parti pris des périphéries. Les "Prosateurs français contemporains" des éditions Rieder (1921-1939), Préface de Valérie Tesnière, Bruxelles, Le Cri, 2007 («CielUlb-Ulg»), pp. 264.

1 Proseguendo nell'iniziativa di far luce sull'esperienza compiuta dalla parigina maison d'édition Rieder negli Anni Venti e Trenta del Novecento, la Gnocchi, con precisione e ordine, raccoglie ed espone il materiale che si trova al centro della collana dei «Prosateurs français contemporains». In base soprattutto a documenti di Archivio e alle lettere di Jean-Richard Bloch, primo direttore dei Pfc, del catalogo l'A. esamina «le contexte, les auteurs, les livres et ce qu'ils signifient» (p. 15): ma l'interesse per la maison si riferisce innanzitutto all'«aventure intellectuelle» piuttosto che all'«entreprise commerciale» (p. 16).

2 La ricerca, che si snoda in dieci capitoli, ognuno dei quali termina in una conclusion, distingue da un lato la produzione dei testi e dall'altro la loro ricezione, vedendo i due momenti come un percorso graduale, interpretabile in un'ottica di storia letteraria. Trattando alcuni partis pris (ad. es. quello della «franchise», p. 20, della «désobéissance», p. 113, del «court, clair, bon», p. 134), l'A. ci fa comprendere, con opportuni esempi, le "periferie" avanzate dal titolo. L'importanza assunta dai criteri geografici e sociali nelle scelte di Rieder è tale che, «dans une formule concise, on pourrait dire que ses auteurs écrivent ailleurs, l'ailleurs, et de préférence autrement» (p. 
116). Gnocchi nota anche i rapporti coi milieux letterari della «Nouvelle Revue française», di «Europe» e del «Mercure de France»e, in questa ricostruzione dell'ambiente in cui vissero gli scrittori, non trascura di segnalare le cause che hanno portato alla "riuscita debole" di un progetto volto a rivendicare un «métissage culturel complexe» (p. 216), indotto dall'apertura all'Altro in termini di «rapprochement des classes par le biais d'une éducation populaire partagée» (p. 69) e di «croisements de langues et de cultures» (p. 173). Dalla Conclusion (pp. 213-218) apprendiamo che occorrerebbe studiare molti altri coins del mondo di Rieder, in modo da sottolinearne ulteriormente il ruolo nell'esperienza del dibattito culturale dell'epoca e nell'ambito della storia editoriale, vera e propria duplice invitation au voyage cui sembra credere anche Valérie Tesnière nelle pagine di prefazione (pp. 7-12). Il testo, che si apre con una chiara Introduction (pp. 13-17), è poi chiuso da due utili Annexes, rispettivamente «I. Table des "Prosateurs français contemporains", par ordre alphabétique», e «II. "Prosateurs français contemporains": chronologie des parutions», oltre che da un Choix bibliographique e da un Index dei nomi. 